\title{
MILLED MICROMOLDS FOR LARGE AREA ALL-ELASTOMER "ROBOT SKIN" WITH 3-AXIS TACTILE SENSING VIA CONTACT RESISTANCE
}

\author{
Alexi Charalambides and Sarah Bergbreiter
}

Department of Mechanical Engineering and the Institute for Systems Research

University of Maryland, College Park, USA

\begin{abstract}
This work demonstrates the first large area, all-elastomer "robot skin" capable of 3-axis tactile sensing, and features a rapid fabrication process and novel taxel geometry. The milling-based fabrication process avoids clean room time while producing features over multiple length scales, from 10 's of microns to 10 's of centimeters, and molds all-elastomer materials to create a mechanically flexible skin. In the presented design, normal force range and resolution were found to be $5 \mathrm{~N}$ and $1 \mathrm{~N}$, respectively, and shear force range and resolution were found to be $430 \mathrm{mN}$ and $100 \mathrm{mN}$, respectively. These values can be tuned via taxel geometry. Cyclic shear force testing was also conducted and no hysteresis was observed up to 100 cycles.
\end{abstract}

\section{INTRODUCTION}

As the field of robotics progresses towards autonomy, advanced tactile sensors are pivotal in enabling safe and dexterous interaction between a robot and its environment [1]. Robotic tasks that generally rely on vision alone, such as grasping, are greatly enhanced with the addition of tactile sensing [2]. Shear force sensing in addition to normal force sensing is especially important in detecting incipient slip of a grasped object [3]. Other wearable systems such as exoskeletons [4], shoes [5, 6], and gloves [7, 8] also stand to benefit from sensor rich "robot skins" that provide real-time force vectors over a large area.

Over the past three decades, notable progress has been made in the field of tactile sensing. Camera-based tactile sensors, in which a soft material is pressed and the deformation is processed visually, have been able to achieve microscale spatial resolution but they're typically limited to a small sensing area and have large, specialized hardware [9]. More compact and versatile sheets of tactile sensor arrays have also been developed [10], and leverage MEMS manufacturing to create microscale sensor geometries essential to multiaxis sensing. However, this method typically results in laborious and complicated multilayer assembly with sub newton force ranges [11]. MEMS manufacturing also limits the sensing area to less than that of a silicon wafer [12]. Other tactile sensors which have large sensing areas have been limited to normal force sensing only $[13,14]$, or have had limited flexibility [15]. Therefore, there is a need for a flexible, large area tactile sensor array capable of shear force sensing in addition to normal force sensing.

\section{SENSOR ARCHITECTURE}

Flexible tactile sensor arrays typically utilize parallel-plate style capacitors [16], or piezoresistive serpentines or strips to detect applied loads [17]. Prior work demonstrated an all-elastomer capacitive tactile sensor with high dynamic range in three axes [10], but required femtofarrad resolution which was difficult to reliably achieve even with proper electrical shielding. Meanwhile, elastomer-based piezoresistive sensors tend to suffer from mechanical hysteresis [18]. A new sensor modality was needed to be more applicable in the field, while avoiding sensor hysteresis.

A contact resistance sensing technique was developed in which two conductive features, referred to as the "pillar" and "pad", come into physical contact as loads are applied, Fig. 1. As a

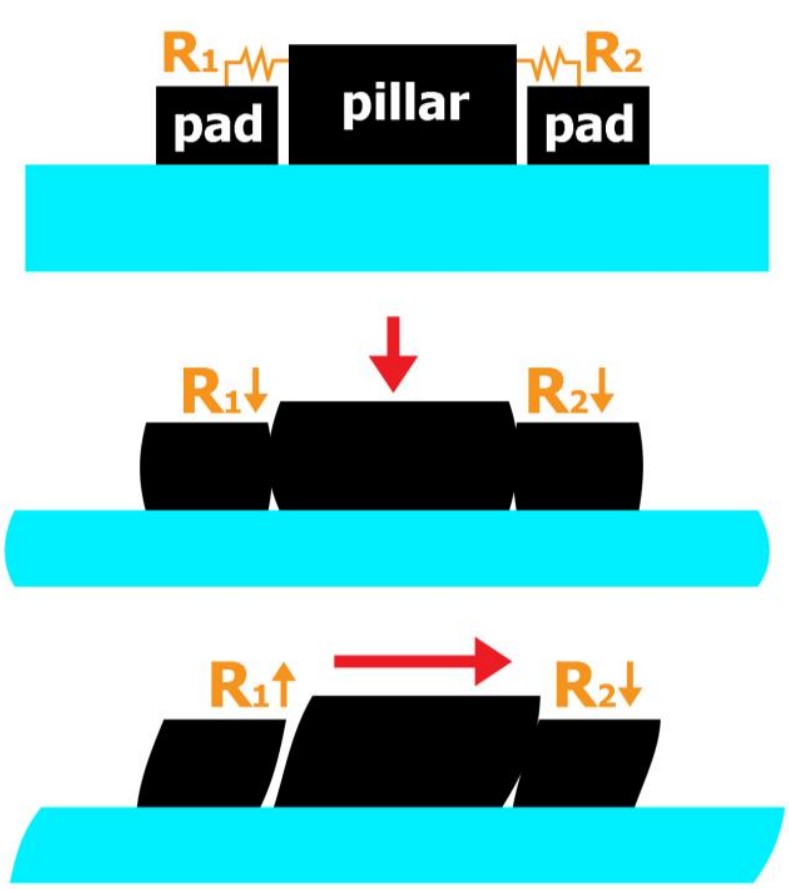

Figure 1: 3-axis force sensing architecture. An applied load causes adjacent features to come into contact with one another, causing predictable changes in contact resistance in both normal and shear directions.

normal force is applied, the pillar and pads flatten and expand through Poisson's effect, and come into contact causing a uniform decrease in contact resistance on each side. Meanwhile, a shear force results in a differential contact resistance; contact resistance decreases in the direction of shear and increases on the opposite side. Elastomers such as polydimethylsiloxane (PDMS) would be especially favorable for this architecture since they are incompressible (Poisson's ratio near 0.5), which maximizes lateral expansion under normal deformation.

\section{FABRICATION}

Computerized numerical control (CNC) milling and micromachining have been widely used to fabricate lab-on-a-chip devices [19, 20], PDMS microstructures and adhesives [21, 22], and even pneumatic logic circuits [23]. In this work, a milling process to cast a conductive elastomer was developed to achieve microscale features over a large area, Fig. 2. This was preferred to clean room fabrication from prior work [10] due to being larger in area and significantly less resource intensive with respect to both time and money. For example, clean room work requires the outsourcing of masks for photolithography, expensive machines and chemicals, and many hours of processing time by a highly trained individual all while being limited to the working area/volume of a silicon wafer. Meanwhile, with the developed manufacturing process it takes less than a day to go from concept to in-hand and ready for testing without sacrificing microscale features. 

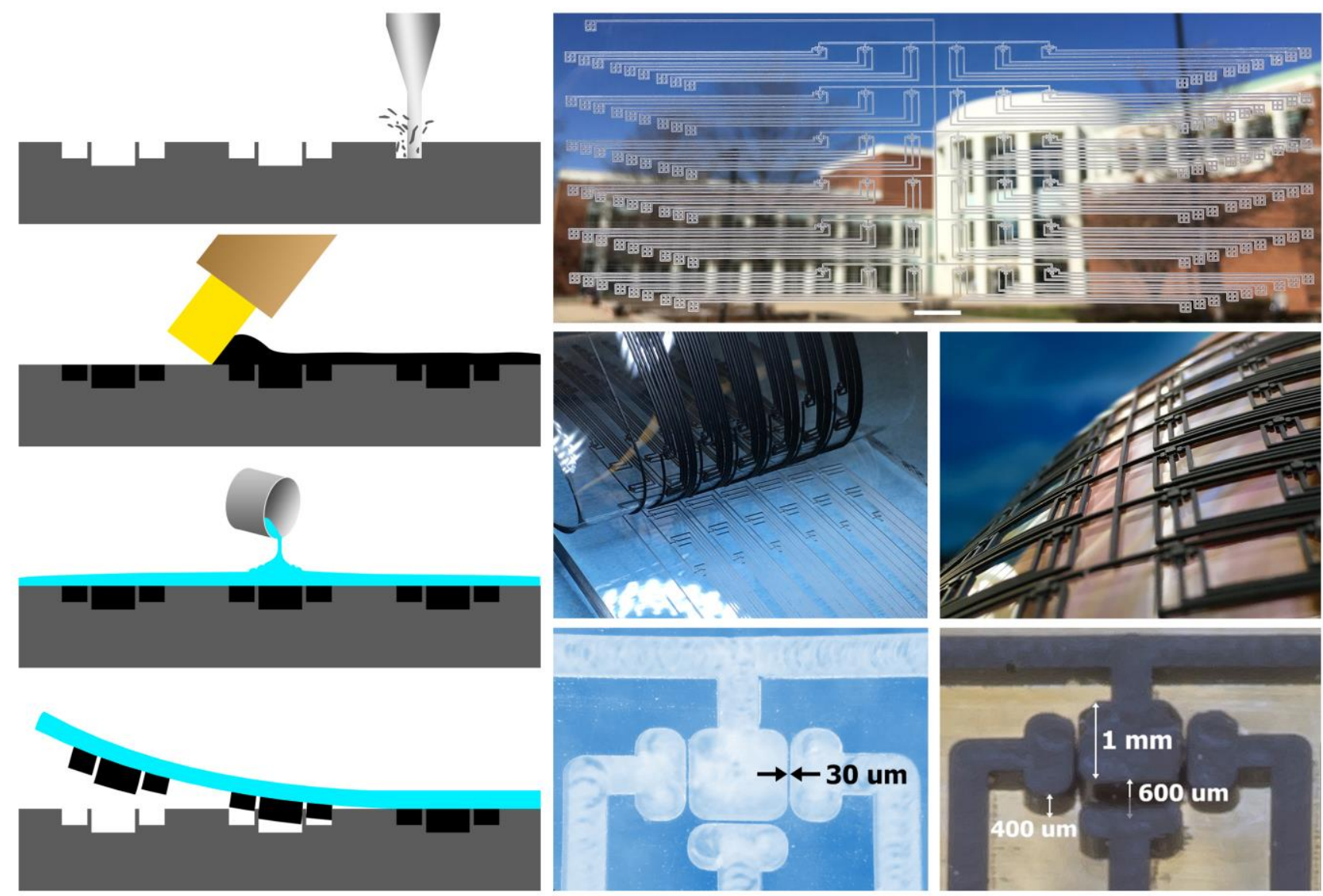

Figure 2: [Left] Fabrication flow chart. Acrylic is milled, refilled with CNT/PDMS, coated with PDMS, and peeled from mold. [Top] Milled acrylic mold. Scale bar is $1 \mathrm{~cm}$. [Center] Robot skin being peeled from the mold. [Right] Isometric view of the robot skin. [Bottom] Close-up of a single taxel in the acrylic mold. [Bottom Right] Close-up of a single taxel after peeling from the mold.

A stock of Acrylic (McMaster-Carr, 8560K355) was milled in a Roland Modela ProII MDX-540 using a $406 \mu \mathrm{m}$ diameter endmill (Microcut USA, 82016), which had a workspace of approximately 12 in by 16 in. NC instructions were coded in Tool Path Language, and generated using CAMotics 1.0.0. No rough cutting for planarizing purposes was necessary as seen in other work [23]; the stock was sufficiently planar as received. Instead, it was mounted in the $\mathrm{CNC}$ machine and leveled by a manual procedure: trenches $0 \mu \mathrm{m}, 100 \mu \mathrm{m}, 200 \mu \mathrm{m}$, and $300 \mu \mathrm{m}$ deep were milled in the four corners of the stock followed by minor adjustments until each corner exhibited three trenches. The stock was cut at $10 \mathrm{~mm} / \mathrm{min}$ at $7000 \mathrm{rpm}$ in taxel areas, and $80 \mathrm{~mm} / \mathrm{min}$ at 10,000 rpm elsewhere, and finished in approximately 2-3 hours. In the presented design, this method produced features that were $400 \mu \mathrm{m}$ and $600 \mu \mathrm{m}$ deep, had a minimum size of $30 \mu \mathrm{m}$, and created an array of 6 by 6 taxels spaced every $1 \mathrm{~cm}$. The total area of the mold was 7 in by 4 in.

The mold was refilled with a conductive elastomer. Carbon nanotubes (Cheap Tubes, 030203) and 10:1 PDMS (Dow Corning, Sylgard 184) were mixed at a total weight percent of 7 wt.\% carbon nanotubes in a centrifugal mixer (Thinky, ARE-310) at $2000 \mathrm{rpm}$ for $90 \mathrm{sec}$. CNT's were found to be favorable over other particles, such as carbon black and silver nanopowder, due to exhibiting excellent mechanical and electrical properties in PDMS with high repeatability. The resulting tar-like CNT/PDMS mixture was spread over the mold and planarized using a screen printing squeegee (Ryonet). 10:1 PDMS was then poured over the mold, placed in vacuum for $20 \mathrm{~min}$ to remove air bubbles, and cured on a

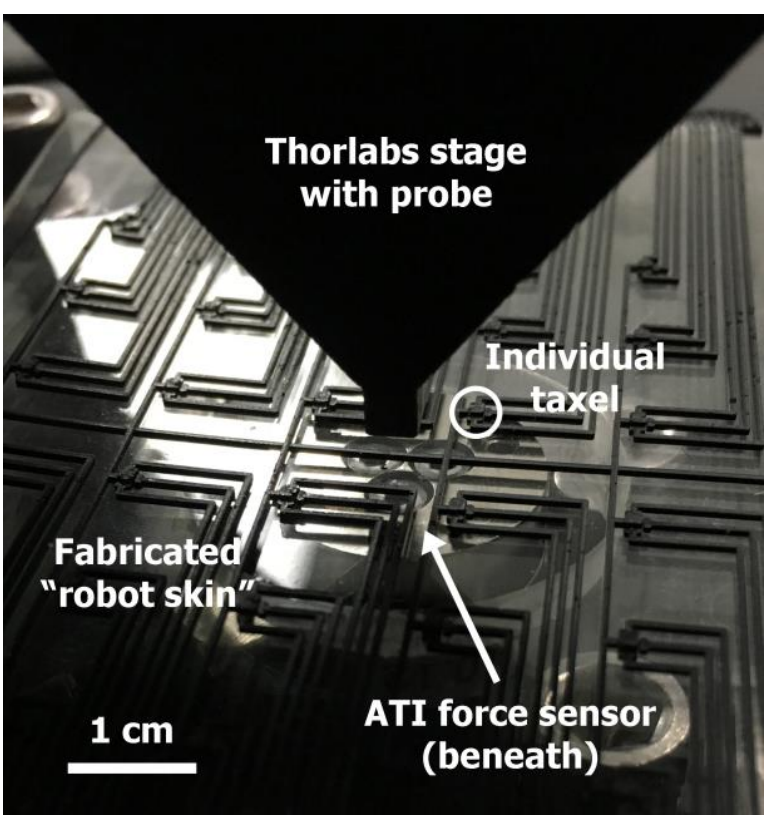

Figure 3: Test setup to characterize the robot skin in 3 axes. A Thorlabs stage equipped with a square probe was used to apply displacements to a taxel, and the resultant forces were read with an ATI force sensor. Voltages were collected with an Arduino Uno (not pictured). 

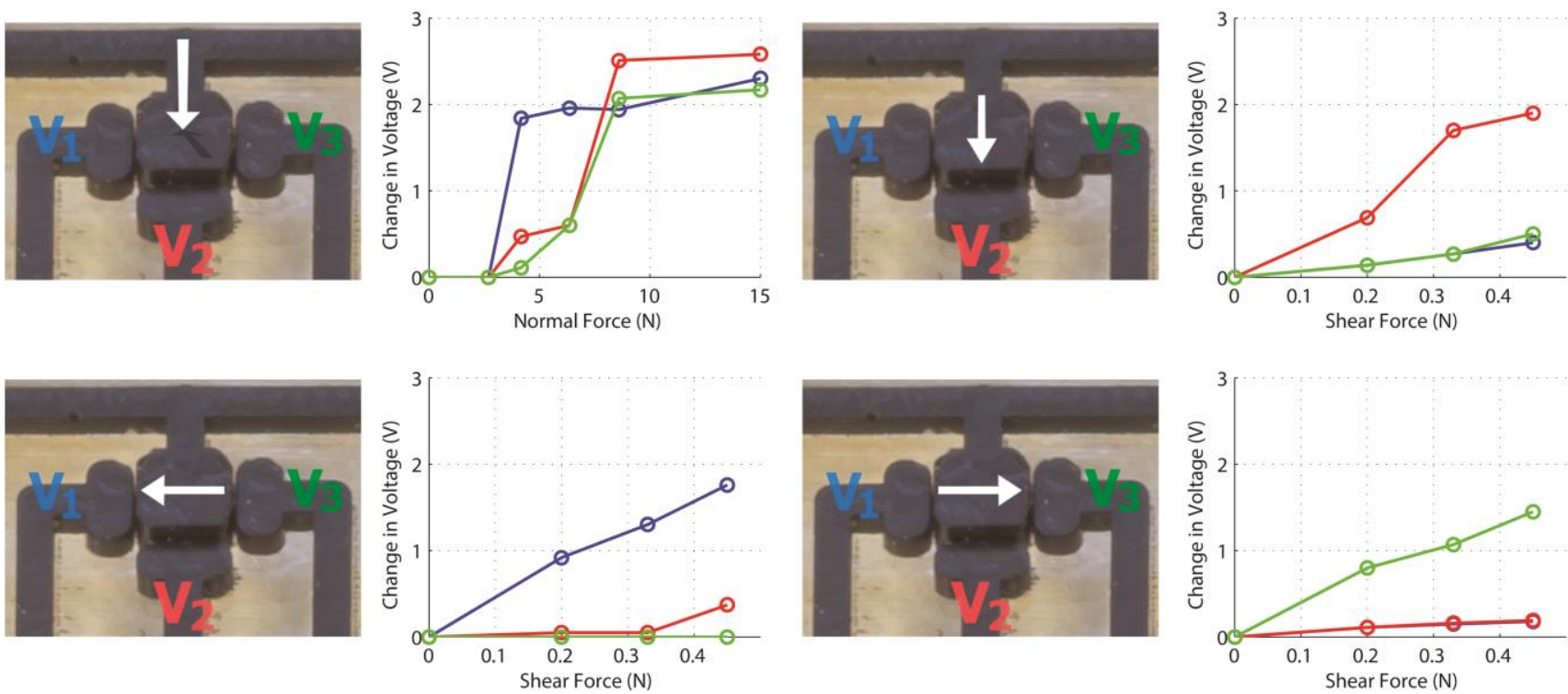

Figure 4: Normal and shear force testing of a single taxel. Each curve corresponds to a single pad of the taxel. In shear force tests, a light normal force of $1 \mathrm{~N}$ was applied prior to shearing to improve contact between the test probe and taxel.
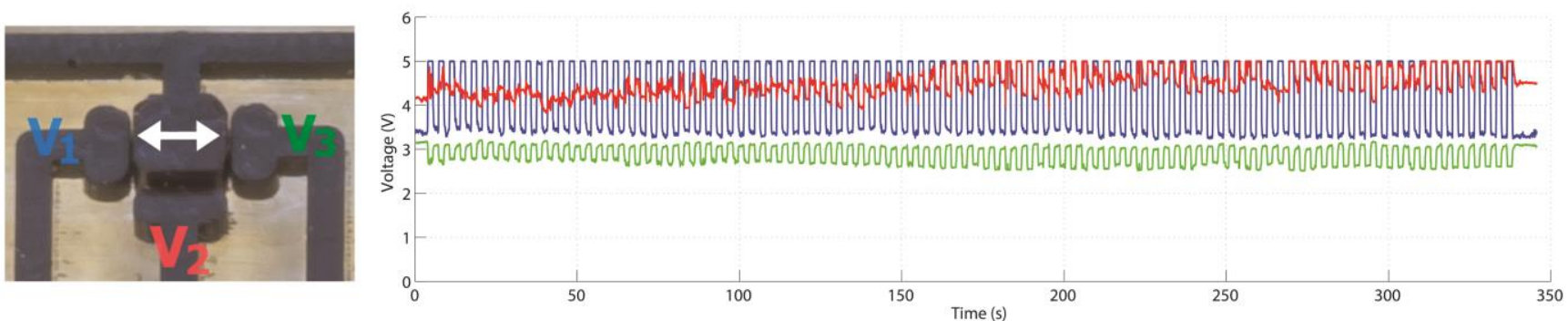

Figure 5: Cyclic shear force testing of a single taxel up to 100 cycles. A normal force of $5 \mathrm{~N}$ was applied before cyclic loading.

hotplate at $160^{\circ} \mathrm{C}$ for $60 \mathrm{~min}$. Lastly, the entire robot skin was peeled from the mold, which can be reused further saving time and money.

Each taxel consisted of one pillar and three adjacent pads, with nominal gaps of $30 \mu \mathrm{m}$ between the pillar and pads. Four pads could not be accommodated due to the space requirements of the electrical routing to the pillar. The total robot skin thickness was $980 \mu \mathrm{m}$, with a PDMS layer thickness of approximately $380 \mu \mathrm{m}$. The completed mold and robot skin can be seen in Fig. 2.

\section{ROBOT SKIN CHARACTERIZATION}

Sensor characterization was done using a Thorlabs PT3-Z8 3 -axis stage equipped with a 3 by $3 \mathrm{~mm}$ Delrin probe, and an ATI Nano17 force sensor with 3 -axis sensing, Fig. 3. A voltage divider was constructed to measure taxel voltages (i.e.: contact resistances). 3-axis force testing was conducted on a single taxel, as well as cyclic testing up to 100 cycles.

As a normal force was applied, a decrease in voltage was observed across all three pads as intended, Fig. 4. The taxel was unresponsive below $3 \mathrm{~N}$, saturated above $8 \mathrm{~N}$, and had a resolution of approximately $1 \mathrm{~N}$. This was because below $3 \mathrm{~N}$ the pillar and pads are not yet in contact, while above $8 \mathrm{~N}$ the sensor can compress no further. The range can be tuned by adjusting the pillar height, pad height, and gap between pillar and pads. This force range is still useful for robotic manipulation applications [24].

Shear forces were applied in the direction of each pad, Fig. 4. A small normal force of $1 \mathrm{~N}$ was applied before shearing to improve contact between the test probe and the taxel, while minimizing the influence of normal force on the results. No change in voltage was observed after applying this normal force. In each shear case, a decrease in voltage was observed across the intended pad, while the voltage of the other pads remained relatively unchanged. Shear force range and resolution were approximately $430 \mathrm{mN}$ and $100 \mathrm{mN}$, respectively. Above $430 \mathrm{mN}$ of shear force slip was observed so the sensor couldn't be tested at higher forces.

Cyclic shear force testing was conducted by applying a normal force of $5 \mathrm{~N}$ followed by loading and unloading of approximately $400 \mathrm{mN}$ of shear force towards $\mathrm{V}_{3}$, Fig. 5 . The pad in the direction of loading decreased in voltage while the opposite increased in voltage, as intended. The magnitude of the increase in voltage was higher than the decrease due to the pad coming out of contact with pillar. No hysteresis was observed after 100 cycles. However, the out of plane pad, $\mathrm{V}_{2}$, experienced a slight increase in voltage. This may have been due to a slight decrease in the measured normal force, from $5 \mathrm{~N}$ to $4.8 \mathrm{~N}$, as the experiment carried on and the taxel underwent stress relaxation.

A 3D-printed rectangular block (Veroblue, RGD840), weighted with a $1 \mathrm{~kg}$ mass, was applied to the robot skin and voltages were collected across the entire 6 by 6 array, Fig. 6 . Change in voltage was calculated after averaging the voltages of each pad, and the largest changes in voltage were localized in the expected area. A modest amount of variance in voltage was observed in the area of the block, and may be due to the variance in taxel quality across the robot skin. Improving the overall yield and quality of individual taxels across the entire robot skin is a subject of current work. 


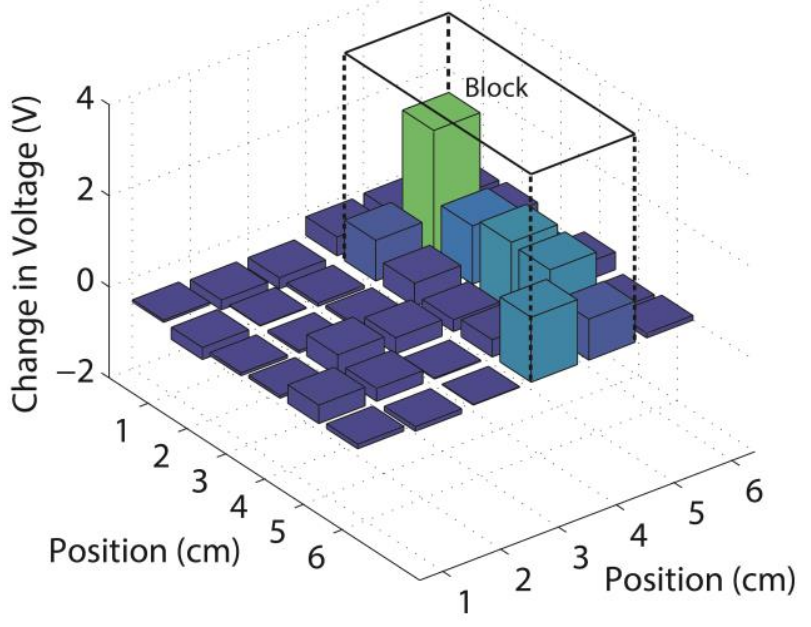

Figure 6: Spatial data of a $1 \mathrm{~kg}$ block resting on the robot skin. Large variations in data may be caused by the quality variation between individual taxels.

\section{LIMITATIONS}

Although affordable manufacturing of large area robot skins with 3-axis sensing and simple electronics has been demonstrated, the most significant drawback was dynamic range. This can be partly mitigated by tuning the taxel geometry, but still lacks the dynamic range of some previous 3 -axis sensors $[10,25]$. To improve dynamic range, the sensing area could be encapsulated with PDMS and capacitive sensing could be used similar to previous work, but at the cost of simple electronics [10]. The current dynamic range is still useful for applications such as grasping, and demonstrating this is a subject of current work. It was also found that at high normal forces (above $8 \mathrm{~N}$ ), the taxels became relatively insensitive to shear forces as the compressed sensor could not deform further; this is an inherent limitation of the design.

During fabrication, a high amount of force is applied to the acrylic mold as the CNT/PDMS is planarized by hand. During this step, it was found that small gaps tend to break. With a gap of 30 $\mu \mathrm{m}$, yield was estimated at $80-90 \%$. In the future, larger gaps could be fabricated to improve yield while also increasing the normal force range, or a more delicate planarization process could be developed.

The sensor architecture left the sensing elements exposed to the environment, which could potentially lead to damage from repeated use. Also, conductive objects such as metals were not compatible with this architecture since they created an electrical short between the pillar and pads. A patterned insulating layer could be placed on top of the robot skin to mitigate this.

Electrical routing was fabricated in the same plane as the sensors, limiting the taxel areal density. However, taxel density can increase if a smaller array (ex: 3 by 3 with a spacing of $3 \mathrm{~mm}$, ideal for fingertips) is desired because the amount of routing is significantly less. A smaller diameter endmill for the routing could also be used. An alternate approach would be to implement a multilayer design that bonds two fabricated layers: a sensing layer and an electrical routing layer. This could also potentially enable each pillar to be surrounded by four pads rather than three.

\section{CONCLUSION}

This work presented a rapid and affordable manufacturing process based on CNC milling, and featured a novel 3-axis tactile sensor architecture using contact resistance. A large area elastomeric robot skin was fabricated with a 6 by 6 array of taxels spaced $1 \mathrm{~cm}$ apart. The fabrication process produced features as small as $30 \mu \mathrm{m}$ without the need of a clean room. Dynamic range was approximately 5:1 in the shear and normal directions, but still had a force range and resolution relevant to robotic manipulation applications. This work may help accelerate the pace of elastomer MEMS research, and result in new conductive elastomeric sensors.

\section{ACKNOWLEDGEMENT}

This material is based upon work supported by NASA under award \#NNX12AM02G. This work is patent pending.

\section{REFERENCES}

[1] Hanna Yousef et. al. Sensors and Actuators A: physical, 167(2):171-187, 2011.

[2] Rui Li et. al. IROS 2014, pg. 3988-3993.

[3] Barrett Heyneman et. al. IJRR, 2015.

[4] Aaron M Dollar et. al. IEEE Transactions on Robotics, 24(1):144-158, 2008.

[5] Stacy J Morris et. al. EMBS/BMES, 2002. Vol. 3, pg. 24682469.

[6] Christian Liedtke et. al. Gait \& posture, 26(1):39-47, 2007.

[7] Dustyn P Roberts et. al. ICRA, 2012, pg. 1520-1525.

[8] Sarah Walsh et. al. 2015.

[9] Eric V Eason et. al. Bioinspiration \& biomimetics, 10(1):016013, 2015.

[10] Alexi Charalambides et. al. JMM, 25(9):095009, 2015.

[11] Hyung-Kew Lee et. al. JMM, 21(3):035010, 2011.

[12] Cheng-Wen Ma et. al. Sensors and Actuators A: Physical, 2014.

[13] Yoshiyuki Ohmura et. al. ICRA 2006, pg. 1348-1353.

[14] Vincent Duchaine et. al. ICRA 2009, pg. 3676-3681.

[15] Giorgio Cannata et. al. MFI 2008, pg. 434-438.

[16] Ming-Yuan Cheng et. al. Sensors, 10(11):10211-10225, 2010.

[17] Soonjae Pyo et. al. JMM, 24(7):075012, 2014.

[18] Letizia Ventrelli et. al. ROBIO 2009, pg. 123-128.

[19] Andreas E Guber et. al. Chemical Engineering Journal, 101(1):447-453, 2004.

[20] Kosuke Iwai et. al. Lab on a Chip, 14(19):3790-3799, 2014.

[21] Paul Day et. al. JMNM, 1(1):011001, 2013.

[22] Dario Carugo et. al. Biomedical microdevices, 18(1):1-10, 2016.

[23] Philip N Duncan et. al. Lab on a Chip, 15(5):1360-1365, 2015.

[24] Robert D Howe. Advanced Robotics, 8(3):245 \{261, 1993.

[25] Chih-Fan Hu et. al. JMM, 21(11):115012, 2011.

\section{CONTACT}

acharala@umd.edu and sarahb@umd.edu 